\title{
Publications de Jacques Barrau sur l'Océanie
}

\section{Catherine Hoare}

\section{(2) OpenEdition}

Journals

Édition électronique

URL : http://journals.openedition.org/jso/1357

DOI : 10.4000/jso.1357

ISSN : 1760-7256

Éditeur

Société des océanistes

Édition imprimée

Date de publication : 1 décembre 2002

Pagination : 15-21

ISSN : 0300-953x

\section{Référence électronique}

Catherine Hoare, «Publications de Jacques Barrau sur l'Océanie », Journal de la Société des Océanistes [En ligne], 114-115 | Année 2002, mis en ligne le 26 mai 2008, consulté le 22 mars 2021. URL : http:// journals.openedition.org/jso/1357 ; DOI : https://doi.org/10.4000/jso.1357

\section{(c) (i) (9)}

Journal de la société des océanistes est mis à disposition selon les termes de la Licence Creative Commons Attribution - Pas d'Utilisation Commerciale - Pas de Modification 4.0 International. 


\title{
Publications de Jacques Barrau sur l'Océanie *
}

\author{
réunies par
}

Catherine HOARE **

1949

Classification et utilisation des terres de la Nouvelle-Calédonie, Proceedings of the 7th Pacific Science Congress, Auckland-Christchurch, $6: 85-88$.

\section{0}

Le Collège d'Agriculture de Gaton au Queensland, Revue Agricole de la NouvelleCalédonie, 1 (1/2) : 9-10, Nouméa: Chambre d'Agriculture de Nouvelle-Calédonie.

Conservation des pommes de terre, Revue Agricole de la Nouvelle-Calédonie, 1 (3/4) : 6-7, Nouméa : Chambre d'Agriculture de NouvelleCalédonie.

Un parasite de l'herbe à oignons, Revue Agricole de la Nouvelle-Calédonie, 1 (3/4) : 7, Nouméa: Chambre d'Agriculture de NouvelleCalédonie.

Méthodes simples de l'appréciation de la valeur d'un sol, Revue Agricole de la NouvelleCalédonie, 1 (5/6) : 9-10, Nouméa: Chambre d'Agriculture de Nouvelle-Calédonie.

La lutte contre les sauterelles en 1950 sur la côte ouest de la Nouvelle-Calédonie, Revue Agricole de la Nouvelle-Calédonie, 1 (7/8) : 4-5, Nouméa: Chambre d'Agriculture de NouvelleCalédonie.

Une plante médicinale de la NouvelleCalédonie : Duboisia myoporoides R. Br., Revue Agricole de la Nouvelle-Calédonie, 1 (7/8): 13-14, Nouméa: Chambre d'Agriculture de Nouvelle-Calédonie.
$\mathrm{Au}$ sujet des secours aux victimes des calamités agricoles, Revue Agricole de la NouvelleCalédonie, 1 (9/10) : 2-3, Nouméa: Chambre d'Agriculture de Nouvelle-Calédonie.

La lutte contre les rats en Nouvelle-Calédonie, Revue Agricole de la Nouvelle-Calédonie, 1 (11/12) : 4-6, Nouméa : Chambre d'Agriculture de Nouvelle-Calédonie.

Sachez reconnaître les rats de NouvelleCalédonie, Revue Agricole de la NouvelleCalédonie, 1 (11/12): 6, Nouméa: Chambre d'Agriculture de Nouvelle-Calédonie.

La maïs hybride, Revue Agricole de la Nouvelle-Calédonie, 1 (11/12): 8-9, Nouméa : Chambre d'Agriculture de Nouvelle-Calédonie.

Une plante fourragère précieuse : le Théosinthe, Revue Agricole de la Nouvelle-Calédonie, 1 (11/12) : 10, Nouméa : Chambre d'Agriculture de Nouvelle-Calédonie.

Liste préliminaire de plantes économiques de la Nouvelle-Calédonie, Document technique $n^{\circ} 6$, Nouméa : Commission du Pacifique Sud, 31 p. [version anglaise : Preliminary list of economic plants in New Caledonia, Technical Paper $n^{\circ} 6$, Nouméa : South Pacific Commission, 31 p.].

avec Bourgeors (J.) - Le blé en NouvelleCalédonie, Revue Agricole de la NouvelleCalédonie, 1 (1/2) : 5-8, Nouméa: Chambre d'Agriculture de Nouvelle-Calédonie.

avec Straatmans (W.) - Premières observations sur les sols de la Nouvelle-Calédonie, Revue Agricole de la Nouvelle-Calédonie, 1 (9/10) : 7-12, Nouméa : Chambre d'Agriculturede Nouvelle-Calédonie.

* Cette bibliographie n'est pas exhaustive mais recense les principales publications de Jacques Barrau sur l'Océanie.

** Laboratoire d'Ethnobiologie-Biogéographie, Muséum national d'Histoire naturelle, Paris. 
1951

Quelques observations sur la végétation, les sols et les climats agricoles de la NouvelleCalédonie, Revue Agricole de la NouvelleCalédonie, 2 (1/2) : 3-8, Nouméa: Chambre d'Agriculture de Nouvelle-Calédonie.

La lutte contre les mauvaises herbes et les plantes introduites envahissantes en NouvelleCalédonie, Revue Agricole de la NouvelleCalédonie, 2 (3/4) : 3-6, Nouméa: Chambre d'Agriculture de Nouvelle-Calédonie.

Premières observations sur les guanos de la Ouamenie, Revue Agricole de la NouvelleCalédonie, 2 (3/4) : 8-9, Nouméa: Chambre d'Agriculture de Nouvelle-Calédonie.

Pour une meilleure qualité des cafés néocalédoniens, Revue Agricole de la NouvelleCalédonie, 2 (5/6): 2, Nouméa: Chambre d'Agriculture de Nouvelle-Calédonie.

Le problème agricole néo-calédonien, Revue Agricole de la Nouvelle-Calédonie, 2 (7/8) : 2-12, Nouméa : Chambre d'Agriculture de NouvelleCalédonie.

Le cerf est-il, en Nouvelle-Calédonie, un facteur d'érosion des sols? Revue Agricole de la Nouvelle-Calédonie, 2 (9/10): 8-9, Nouméa: Chambre d'Agriculture de Nouvelle-Calédonie.

Coopération et syndicalisme, Revue Agricole de la Nouvelle-Calédonie, 2 (11/12), Nouméa : Chambre d'Agriculture de Nouvelle-Calédonie.

avec Cathala (René). - Sols et agriculture aux Îles Gilbert, Revue Agricole de la NouvelleCalédonie, 2 (5/6) : 6-11, Nouméa: Chambre d'Agriculture de Nouvelle-Calédonie.

\section{2}

Humus et fertilité, Revue Agricole de la Nouvelle-Calédonie, 3 (1/2) : 3-4, Nouméa : Chambre d'Agriculture de Nouvelle-Calédonie.

À propos de la motoculture, Revue Agricole de la Nouvelle-Calédonie, 3 (3/4) : 2-4, Nouméa : Chambre d'Agriculture de Nouvelle-Calédonie.

À propos de la conservation du sol, Revue Agricole de la Nouvelle-Calédonie, 3 (7/8) : 2-3, Nouméa : Chambre d'Agriculture de NouvelleCalédonie.

Notes de botanique économique néocalédonienne: 1. Citrus macroptera Montr., oranger de la Nouvelle-Calédonie, Revue Agri- cole de la Nouvelle-Calédonie, 3 (7/8) : 13, Nouméa: Chambre d'Agriculture de NouvelleCalédonie.

Notes de botanique économique néocalédonienne: 2. Les "Magnagnas" de la Nouvelle-Calédonie, Revue Agricole de la Nouvelle-Calédonie, 3 (9/10):8-9, Nouméa : Chambre d'Agriculture de Nouvelle-Calédonie.

Recherches au Queensland en matière d'agriculture et d'élevage tropicaux, Document technique $n^{\circ} 43$, Nouméa: Commission du Pacifique Sud, $88 \mathrm{p}$.

L'utilisation agricole et pastorale des terres de la Nouvelle-Calédonie, Études mélanésiennes, 5 (7) : 1-22, Nouméa : Société d'Études mélanésiennes.

Le cocotier et le caféier en Nouvelle Calédonie, Revue Internationale des Produits Coloniaux et du Matériel Colonial, Paris, 287/288 : 147-149.

Le problème des pâturages de la NouvelleCalédonie, Revue Agricole de la NouvelleCalédonie, 4 (7/8) : 3-10, Nouméa: Chambre d'Agriculture de Nouvelle-Calédonie.

Agriculteurs et éleveurs! Attention! Revue Agricole de la Nouvelle-Calédonie, 4 (7/8) : 18, Nouméa : Chambre d'Agriculture de NouvelleCalédonie.

Recherches sur les pâturages et fourrages au Queensland, Revue Agricole de la NouvelleCalédonie, 4 (7/8) : 11-16, Nouméa : Chambre d'Agriculture de Nouvelle-Calédonie.

\section{4}

Le cocotier en Nouvelle-Calédonie, Revue Agricole de la Nouvelle-Calédonie, 5 (3/4) : 8-10, Nouméa : Chambre d'Agriculture de NouvelleCalédonie.

Un essai pratique d'amélioration des pâturages réalisé par un éleveur de NouvelleCalédonie, Études mélanésiennes, 6 (8) : 56-61, Nouméa : Société d'Études mélanésiennes.

Croquis et notes sur quelques plantes alimentaires utilisées par les autochtones à la NouvelleCalédonie, Études mélanésiennes, 6 (8) : 61-65, Nouméa : Société d'Études mélanésiennes.

Traditional subsistence economy and agricultural progress in: Melanesia, South Pacific Congress Quaterly Bulletin, Noumea: South Pacific Commission, 6 p.

avec Massal (Émile) - Cultures vivrières du Pacifique : l'arbre à pain, Bulletin trimestriel de la Commission du Pacifique Sud, 4 (4) : 68-69, Nouméa : Commision du Pacifique Sud. 
1955

Schematic ecology of the South Pacific region in : Consultations on plant collection and introduction, 53-57, Nouméa.

L'agriculture vivrière mélanésienne, Nouméa : Commission du Pacifique Sud, 206 p.

avec Massal (Émile) - Pacific subsistence crops, Taros, South Pacific Congress Quaterly Bulletin, Noumea : South Pacific Commission, $5 \mathrm{p}$.

\section{6}

L'agriculture vivrière des polynésiens et micronésiens, Nouméa : Commission du Pacifique Sud, 135 p.

L'agriculture vivrière indigène aux NouvellesHébrides, Journal de la Société des Océanistes, 12 : 181-215, Paris : Musée de l'Homme.

L'agriculture vivrière autochtone de la Nouvelle-Calédonie, précédée de L'organisation sociale et coutumière de la population autochtone par Jean Guiart, Document technique $\mathrm{n}^{\mathrm{o}}$ 87, Nouméa : Commission du Pacifique Sud, $153 \mathrm{p}$.

L'arrow root polynésien : Tacca leontopetaloides, (L.) O. Kuntze, La Terre et la Vie, 2 : 80-82, Paris.

Le milieu et l'agriculture traditionnelle en Mélanésie, Annales de Géographie, 65 (351): 362-382, Paris : Librairie Armand Colin.

Quelques plantes alimentaires des rivages marins du Pacifique Sud, La Terre et la Vie, 2 : 77-79, Paris.

Les Légumineuses à tubercules alimentaires de la Mélanésie, La Terre et la Vie, 1: 11-16, Paris.

Plantes alimentaires de base des Mélanésiens, J. d'Agric. Trop. et de Bot. Appl., 3 : 323, Paris : Laboratoire d'Agronomie Tropicale, Muséum National d'Histoire Naturelle.

Les ignames alimentaires des Îles du Pacifique Sud, J. d'Agric. Trop. et de Bot. Appl., 3 : 385, Paris: Laboratoire d'Agronomie Tropicale, Muséum National d'Histoire Naturelle.

Un séchoir pour conserver le fruit de l'arbre à pain, Bulletin trimestriel de la Commission du Pacifique Sud, 7 (2) : 29-30, Nouméa : Commission du Pacifique Sud.

L'agriculture vivrière autochtone de la Nouvelle-Calédonie, J. d'Agric. Trop. et de Bot. Appl., 3 : 906, Paris : Laboratoire d'Agronomie
Tropicale, Muséum National d'Histoire Naturelle.

avec Massal (Émile) - Plantes alimentaires du Pacifique Sud, Document Technique $\mathrm{n}^{\circ} 94$, Nouméa : Commission du Pacifique Sud, 91 p. [version anglaise : Food plants of the South Sea Islands, Technical Paper $n^{\circ}$ 94, Nouméa : South Pacific Commission, 91 p.].

avec Massal (Émile). — Cultures vivrières du Pacifique : le cocotier, Bulletin trimestriel de la Commission du Pacifique Sud, 7 (2) : 12-19, Nouméa : Commision du Pacifique Sud.

\section{7}

Les Aracées à tubercules alimentaires des Îles du Pacifique Sud, J. d'Agric. Trop. et de Bot. Appl., 4 : 34, Paris : Laboratoire d'Agronomie Tropicale, Muséum National d'Histoire Naturelle.

L'Arbre à pain en Océanie, J. d'Agric. Trop. et de Bot. Appl., 4: 117, Paris: Laboratoire d'Agronomie Tropicale, Muséum National d'Histoire Naturelle.

À propos du Piper methysticum Forster, J. d'Agric. Trop. et de Bot. Appl., 4 : 270-273, Paris : Laboratoire d'Agronomie Tropicale, Muséum National d'Histoire Naturelle.

Usage curieux d'une Aracée de la NouvelleGuinée, J. d'Agric. Trop. et de Bot. Appl., 4: 348-349, Paris : Laboratoire d'Agronomie Tropicale, Muséum National d'Histoire Naturelle.

Les toupies des Buang de la Nouvelle-Guinée, J. d'Agric. Trop. et de Bot. Appl., 4 : 350-351, Paris: Laboratoire d'Agronomie Tropicale, Muséum National d'Histoire Naturelle.

Le Duboisia myoporoides R. Br., plante médicinale de la Nouvelle-Calédonie, J. d'Agric. Trop. et de Bot. Appl., 4: 453, Paris : Laboratoire d'Agronomie Tropicale, Muséum National d'Histoire Naturelle.

The «Bush fallowing » system of cultivation in the continental Islands of Melanesia: The Proceedings of the Ninth Pacific Science Congress, 7 : 53-55, [Bangkok] : [s.n.].

Les atolls océaniens : essai d'agronomie, Études d'Outre-Mer, août-sept : 253-267, Marseille : Institut Français d'Outre-Mer.

L'énigme de la patate douce en Océanie, Études d'Outre-Mer, avril : 83-87, Marseille : Institut français d'Outre-Mer.

avec Devambez (Louis) - Quelques résultats inattendus de l'acclimatation en Nouvelle- 
Calédonie, La Terre et la Vie, 4 : 324-335, Paris : Société nationale d'Acclimatation.

avec GIRARD (Françoise) - Quelques plantes alimentaires et rituelles en usage chez les Buang (Nouvelle-Guinée), J. d'Agric. Trop. et de Bot. Appl., 4 : 212, Paris : Laboratoire d'Agronomie Tropicale, Muséum National d'Histoire Naturelle.

\section{8}

Subsistence agriculture in Melanesia, Bernice P. Bishop Museum Bulletin, 19, Honolulu : Museum, $111 \mathrm{p}$.

A reconnaissance survey in the field of economic botany in western Melanesia : report on a visit to Netherlands New Guinea, the Territory of Papua \& New Guinea and the British Solomon Islands Protectorate, $31^{\text {st }}$ January- $23^{\text {rd }}$ April, 1958 ; Nouméa : South Pacific Commission, $60 \mathrm{p}$.

Present-day problems in the utilisation of pasture land in New Caledonia, Proceedings of the Eighth Pacific Science Congress, 4 B : 573-576, Quezon City : National Research Council of the Philippines.

Nouvelles observations au sujet des plantes hallucinogènes d'usage autochtone en NouvelleGuinée, J. d'Agric. Trop. et de Bot. Appl., 5 : 377, Paris: Laboratoire d'Agronomie Tropicale, Muséum National d'Histoire Naturelle.

Ethnobotanique et traversées du Pacifique en radeau, J. d'Agric. Trop. et de Bot. Appl., 5 : 665, Paris: Laboratoire d'Agronomie Tropicale, Muséum National d'Histoire Naturelle.

From digging stick to machine-drawn plough, South Pacific Commission Quaterly Bulletin, avril, Nouméa : South Pacific Commission, 3 p.

\section{9}

Marquesas journey, South Pacific Bulletin, $\mathrm{n}^{\circ} 1,6 \mathrm{p}$., Nouméa : South Pacific Commission.

Le fabuleux arbre à pain, Naturalia, $69: 7-13$, Saint-Ouen : Chaix.

Esquisse écologique de la Nouvelle Guinée, La Terre et la Vie, 4 : 291-307, Paris : Société nationale de protection de la nature et d'acclimatation de France.

Sur l'origine et la distribution du bananier Fehi Musa troglodytarum L., Études mélanésiennes, 8/9 (12/13) : 45-49, Nouméa : Société d'études mélanésiennes.
A new approach to coconut selection, South Pacific Congress Quaterly Bulletin, 1, Nouméa : South Pacific Commission, 2 p.

Louis-Antoine de Bougainville et la flore économique de Tahiti, J. d'Agric. Trop. et de Bot. Appl., 6: 115, Paris, Laboratoire d'Agronomie Tropicale, Muséum National d'Histoire Naturelle.

Fruits et graines du Taro, Colocasia esculenta Linné, J. d'Agric. Trop. et de Bot. Appl., $6: 436$, Paris, Laboratoire d'Agronomie Tropicale, Muséum National d'Histoire Naturelle.

The Sago palms and other food plants of Marsh dwellers in the South Pacific Islands, Economic Botany, 13 (2) : 151-162, New York, The New York Botanical Garden.

The bush-fallowing system of cultivation in the continental islands of Melanesia, Proceedings of the 9th Pacific Science Congress : 54-56, Bangkok.

L'agriculture polynésienne au contact avec des étrangers, Journal de la Société des Océanistes, 15 (15) : 147-163, Paris Musée de l'Homme.

avec Maclet (Jean-Noël) - Catalogue des plantes utiles aujourd'hui présentes en Polynésie Française, J. d'Agric. Trop. et de Bot. Appl., 6 (1-2-3) : 1-21 et 161-184, Paris, Laboratoire d'Agronomie Tropicale, Muséum National d'Histoire Naturelle.

\section{0}

Le santal, Bulletin de la Commission du Pacifique Sud, 10 (3) : 19-20, Nouméa.

The Sandalwood tree, South Pacific Bulletin, 4 : 39-41, Nouméa, [s.n.].

The New Hebrides, South Pacific Bulletin, 10 (3) : 22-57, Nouméa, [s.n.].

Plant introduction in the tropical Pacific, its role in economic development, Pacific Viewpoint $1(1): 1-11$.

\section{1}

Subsistence agriculture in Polynesia and Micronesia, Bernice P. Bishop Museum Bulletin, 223, Honolulu, Museum, $94 \mathrm{p}$.

Une réunion technique sur la prospection et l'introduction des plantes, J. d'Agric. Trop. et de Bot. Appl., 8 : 200, Paris, Laboratoire d'Agronomie Tropicale, Muséum National d'Histoire Naturelle. 
1962

Les coprahs français du Pacifique et leur commercialisation, Bulletin de la Société de Géographie de Marseille, 73 (3) : 121-139.

Edible yams of the South Sea Islands, species present, vernacular names and distribution, Proceedings of the Ninth Pacific Science Congress, 4 : 309-311, Bangkok, [s.n.].

Observations et travaux récents sur les végétaux hallucinogènes de la Nouvelle-Guinée, $J$. d'Agric. Trop. et de Bot. Appl., 9 : 245, Paris, Laboratoire d'Agronomie Tropicale, Muséum National d'Histoire Naturelle.

Les plantes alimentaires de l'Océanie : origines, distribution et usages, Annales du Musée Colonial de Marseille, 63e à 69e années, 7e série, Marseille, Faculté des Sciences, 275 p.

Notes on the significance of some vernacular names of food plants in the South Pacific islands, Proceedings of the Ninth Pacific Science Congress, 4 : 296-298, [Bangkok], [s.n.].

À propos de la Nouvelle-Calédonie rurale, Revue du Centre de Productivité et des Études Économiques de la Nouvelle-Calédonie, 3 : 4-11, Nouméa.

Social and economic aspects of the effects of early man's action on the vegetation of the humid tropics, Nouméa, South Pacific Commission : 373-374.

\section{3}

Le Service d'introduction des plantes et d'exploration botanique de la Commission du Pacifique Sud, Comptes rendus d'une réunion technique sur la prospection et l'introduction des plantes, Rome, [s.n.].

L'agriculture des îles Wallis et Futuna, Journal de la Société des Océanistes, 19 : 157-171, Paris, Musée de l'Homme.

Plant exploration and introduction in the improvment of the coconut palm, Proceedings of FAO technical working party on coconut production and processing, Bangkok: United Nation Food and Agricultural Organization.

(comme éditeur), Plants and the migrations of Pacific peoples, a Symposium, Hawaï, 1961, Bishop Museum Press, 136 p.

\section{4}

Notes sur la signification de quelques noms vernaculaires de plantes alimentaires dans les îles du Pacifique Sud, J. d'Agric. Trop. et de Bot. Appl., 11 (5-6-7) : 205-208, Paris, Laboratoire d'Ethnobotanique, Muséum National d'Histoire Naturelle.

\section{5}

Witnesses of the past : notes on some food plants of Oceania, Ethnology, 4, (3) : 282-294, Pittsburgh, University of Pittsburgh.

Quelques notes à propos de plantes utiles des hautes terres de la Nouvelle-Guinée, J. d'Agric. Trop. et de Bot. Appl., 12 : 44, Paris, Laboratoire d'Ethnobotanique, Muséum National d'Histoire Naturelle.

Note sur la fabrication du tissu d'écorce dit Tapa, à l'île Uvéa encore appelée Wallis, $J$. d'Agric. Trop. et de Bot. Appl., 12 : 211, Paris, Laboratoire d'Ethnobotanique, Muséum Natio nal d'Histoire Naturelle.

Histoire et préhistoire horticoles de l'Océanie tropicale, Journal de la Société des Océanistes, 21 (21) : 55-78, Paris, Musée de l'Homme.

Gardeners of Oceania, Discovery, 1 (1) : 12-19, New Haven.

avec Bromley (Myron) - Présence d'un Coix cultivé dans les montagnes de la NouvelleGuinée, J. d'Agric. Trop. et de Bot. Appl., 12 (12) : 781-782, Paris, Laboratoire d'Ethnobotanique, Muséum National d'Histoire Naturelle.

\section{6}

Guidelines for a study of medicinal plants used in flok medicine in tropical Oceania, Preliminary report to the South Pacific Commission, [S.1.], [s.n.], 34 p.

An Ethnobotanical guide for anthropological research in Malayo-Oceania (preliminary draft), [S.1.], UNESCO Science Cooperation Office for Southeast Asia, 149 p.

Jardins botaniques et d'essais aux îles françaises de la mer du Sud, Les Cahiers du Pacifique, 9: 15-27, Paris, Muséum National d'Histoire Naturelle.

\section{7}

Les hommes, les plantes et la mer en Océanie tropicale, Les Cahiers du Pacifique, 10 : 59-78, Paris, [s.n.].

De l'homme cueilleur à l'homme cultivateur : l'exemple océanien, Cahiers d'Histoire mondiale, 
10 (2) : 275-292, Neuchatel, Éditions de la Baconnière.

\section{8}

La Nouvelle-Calédonie rurale, Horizons $d u$ monde, numéro spécial 'Nouvelle-Calédonie 68' : 60-69, Paris.

L'humide et le sec, in: Peoples and cultures of the Pacific : an anthropological reader : 113-132, New York, The Natural History Press.

Rapport de mission à l'île Wallis, 26 décembre 1967-12 janvier 1968, Nouméa, [s.n.], 20 p. dactylographiées.

Une tentative de colonisation européenne en Océanie tropicale : les Français à la NouvelleCalédonie, La Revue de Géographie de Montréal, 22 (2) : 187-195, Montréal, Département de géographie, Université de Montréal.

\section{9}

avec Peeters (Alice) - Food processing techniques of Australian collectors and archaic cultivators as possible indicators of the history and prehistory of vegetable food preparation, Providence, Meeting of the North Eastern Anthropological Association, Symposium on anthropology of food and food habits, $11 \mathrm{p}$.

\section{0}

Ethno-botanique spéciale. L'homme et son environnement végétal en région tropicale humide : l'exemple Malayo-océanien, in : Cours de ethno-botanique et ethno-zoologie (19691970), 2: 1-52, Paris, Institut d'Ethnologie, Faculté des Lettres et Laboratoire d'Ethnobotanique et d'Ethno-zoologie, Muséum National d'Histoire Naturelle.

Note sur le langage des plantes en NouvelleCalédonie mélanésienne, J. d'Agric. Trop. et de Bot. Appl., 17: 461-463, Paris, Laboratoire d'Ethnobotanique, Muséum National d'Histoire Naturelle.

The food situation in the Pacific, review and outlook, in: Food problems in Asia and the Pacific, Proceedings of the Seminar held at the East West Center, Honolulu : 12-21.

$\mathrm{La}$ région indo-pacifique comme centre de mise en culture et de domestication des végétaux, J. d'Agric. Trop. et de Bot. Appl., 17 : 487, Paris, Laboratoire d'Ethnobotanique, Muséum National d'Histoire Naturelle.
Plantes utiles de Tahiti, Société des Océanistes (Musée de l'Homme) dossier 9.

L'archipel du paradoxe: présentation de la Nouvelle-Calédonie et des îles voisines, Asie nouvelle, numéro spécial Nouvelle-Calédonie : 7-13, [S.1.], [s.n.].

\section{2}

Caesalpinia crista L., plante médicinale populaire en Mélanésie néo-calédonienne, J. d'Agric. Trop. et de Bot. Appl., 19 : 593, Paris, Laboratoire d'Ethnobotanique, Muséum National d'Histoire Naturelle.

avec O'Reilly (P.) - Jardin botanique de Papeari, Tahiti, Paris Société des Océanistes, Dossier 9, $36 \mathrm{p}$.

avec PeEters (Alice) - Histoire et préhistoire de la préparation des aliments d'origine végétale : les techniques d'utilisation de ces aliments chez les cueilleurs et les cultivateurs archaïques de l'Australasie, Journal de la Société des Océanistes, 28 (35) : 141-152, Paris, Musée de l'Homme.

\section{4}

À propos des Corydalis utiles, J. d'Agric. Trop. et de Bot. Appl., 21: 163, Paris, Laboratoire d'Ethnobotanique, Muséum National d'Histoire Naturelle.

\section{5}

Un taro, un poisson, une papaye : manuel d'éducation alimentaire et de nutrition appliquée à l'usage des éducateurs de l'Océanie tropicale (chap. 37, 38), Commission du Pacifique Sud, Nouméa.

Guide bibliographique: environnements naturels, sociétés humaines et développement en Papouasie-Nouvelle-Guinée, Paris, Unité de documentation et de liaison sur l'écodéveloppement, Maison des Sciences de l'Homme, $71 \mathrm{p}$.

\section{6}

Et le rat des îles océaniennes? Rattus, 3. 
1978

Ecology and evolution of agricultural and pastoral systems of land use in the IndoOceanian savanna environments, including the impact of colonial on indigenous systems, in : Human ecology in savanna environments, Symposium $\mathrm{n}^{\circ} 79$, New York, Wenner-Gren Foundation for Anthropological Research, 18 p.

\section{0}

Indigenous and colonial land-use systems in Indo-Oceania savannas: the case of NewCaledonia, in : Human ecology in Savanna envi- ronment, David D. Harris ed. : 253-265, Londres, Academic Press Inc. Ltd.

\section{9}

Les pérégrinations de l'arbre à pain, Artocarpus utilis (Parkinson) Fosberg, Moracées, Feuille d'Information de la Société des Amis du Muséum et du Jardin des Plantes, déc. : 52-54.

\section{[s.d.]}

Pour une étude des plantes médicinales utilisées en médecine populaire et traditionnelle en Océanie tropicale, [S.1.] : [s.n.], 40 p. 